$\mathrm{J}$ o u r n a l of

Mathematics

and Applications

No 35, pp 39-51 (2012)

\title{
Controllability of the semilinear Benjamin-Bona-Mahony equation
}

\author{
H. Leiva and N. Merentes and J.L. Sanchez
}

Submitted by: Józef Banaś

ABstRACT: In this paper we prove the interior approximate controllability of the following Generalized Semilinear Benjamin-Bona-Mahony type equation (BBM) with homogeneous Dirichlet boundary conditions

$$
\left\{\begin{array}{l}
z_{t}-a \Delta z_{t}-b \Delta z=1_{\omega} u(t, x)+f(t, z, u(t, x)), \quad t \in(0, \tau], \quad x \in \Omega, \\
z(t, x)=0, \quad t \geq 0, \quad x \in \partial \Omega
\end{array}\right.
$$

where $a \geq 0$ and $b>0$ are constants, $\Omega$ is a domain in $\mathbb{R}^{N}, \omega$ is an open nonempty subset of $\Omega, 1_{\omega}$ denotes the characteristic function of the set $\omega$, the distributed control $u$ belongs to $L^{2}\left(0, \tau ; L^{2}(\Omega)\right)$ and the nonlinear function $f:[0, \tau] \times \mathbb{R} \times \mathbb{R} \rightarrow \mathbb{R}$ is smooth enough and there are $c, d, e \in \mathbb{R}$, with $c \neq-1, e a+b>0$, such that

$$
\sup _{(t, z, u) \in Q_{\tau}}|f(t, z, u)-e z-c u-d|<\infty
$$

where $Q_{\tau}=[0, \tau] \times \mathbb{R} \times \mathbb{R}$. We prove that for all $\tau>0$ and any nonempty open subset $\omega$ of $\Omega$ the system is approximately controllable on $[0, \tau]$. Moreover, we exhibit a sequence of controls steering the system from an initial state $z_{0}$ to an $\epsilon$-neighborhood of the final state $z_{1}$ on time $\tau>$ 0 . As a consequence of this result we obtain the interior approximate controllability of the semilinear heat equation by putting $a=0$ and $b=1$.

AMS Subject Classification: Primary 93B05, Secondary 93C25.

Key Words and Phrases: interior controllability, semilinear BBM equation, strongly continuous semigroups

This work was supported by MCTI, CDCHT-ULA under projects ConCiencia-3837, C-1667-0905-AA and by BCV

COPYRIGHT (C) by Publishing Department Rzeszów University of Technology P.O. Box 85, 35-959 Rzeszów, Poland 


\section{Introduction.}

As we pointed out in [11], the original Benjamin-Bona-Mohany Equation is a nonlinear one; even so, in this reference we proved the interior controllability of the linear BBM equation, which is essential for a subsequent study of the nonlinear BBM equation. So, in this paper we shall prove the interior controllability of the following Generalized Semilinear Benjamin-Bona-Mahony type equation (BBM) with homogeneous Dirichlet boundary conditions

$$
\left\{\begin{array}{l}
z_{t}-a \Delta z_{t}-b \Delta z=1_{\omega} u(t, x)+f(t, z, u(t, x)), \quad t \in(0, \tau], \quad x \in \Omega, \\
z(t, x)=0, \quad t \geq 0, \quad x \in \partial \Omega
\end{array}\right.
$$

where $a \geq 0$ and $b>0$ are constants, $\Omega$ is a domain in $\mathbb{R}^{N}, \omega$ is an open nonempty subset of $\Omega, 1_{\omega}$ denotes the characteristic function of the set $\omega$, the distributed control $u \in L^{2}\left(0, \tau ; L^{2}(\Omega)\right)$ and the nonlinear function $f:[0, \tau] \times \mathbb{R} \times \mathbb{R} \rightarrow \mathbb{R}$ is smooth enough and there are $c, d, e \in \mathbb{R}$, with $c \neq-1$, ea $+b>0$, such that

$$
\sup _{(t, z, u) \in Q_{\tau}}|f(t, z, u)-e z-c u-d|<\infty
$$

where $Q_{\tau}=[0, \tau] \times \mathbb{R} \times \mathbb{R}$. Under these conditions we prove the following statement: For all $\tau>0$ and any nonempty open subset $\omega$ of $\Omega$ the system is approximately controllable on $[0, \tau]$. Moreover, we exhibit a sequence of controls steering the system from an initial state $z_{0}$ to an $\epsilon$-neighborhood of the final state $z_{1}$ on time $\tau>0$. As a consequence of this result we obtain the interior approximate controllability of the semilinear heat equation by putting $a=0$ and $b=1$.

We note that, the interior approximate controllability of the linear heat equation

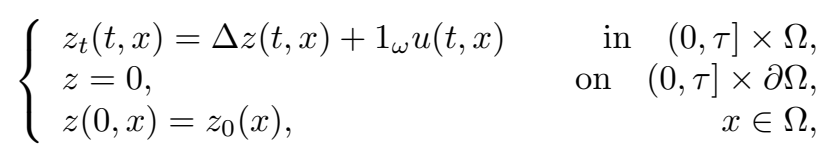

has been study by several authors, particularly by [15],[16],[17]; and in a general fashion in [14].

The approximate controllability of the heat equation under nonlinear perturbation $f(z)$ independents of $t$ and $u$ variables

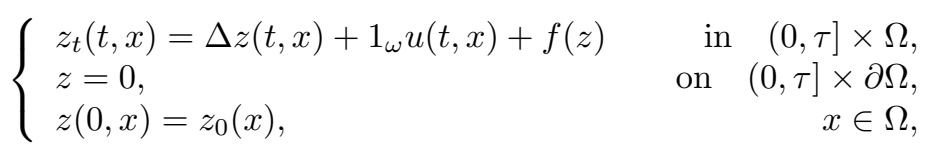

has been studied by several authors, particularly in [6], [7] and [8], depending on conditions impose to the nonlinear term $f(z)$. For instance, in [7] and [8] the approximate controllability of the system (1.4) is proved if $f(z)$ is sublinear at infinity, i.e.,

$$
|f(z)| \leq E|z|+D
$$

Also, in the above references, the authors mentioned that when $f$ is superlinear at the infinity, the approximate controllability of the system (1.4) fails. 
In this paper we use different technique for the linear part (see [14], [11]) and Schauder fixed point Theorem for the semilinear system.

Now, we shall describe the strategy of this work:

First, we observe that the hypothesis (1.2) is equivalent to the existence of $e, c \in \mathbb{R}$, with $c \neq-1$, ea $+b>0$, such that

$$
\sup _{(t, z, u) \in Q_{\tau}}|f(t, z, u)-e z-c u|<\infty
$$

where $Q_{\tau}=[0, \tau] \times \mathbb{R} \times \mathbb{R}$.

Second, we prove that the auxiliary linear system

$$
\left\{\begin{array}{l}
z_{t}-a \Delta z_{t}-b \Delta z=1_{\omega} u(t, x)+e z+c u(t, x), \quad t \in(0, \tau], \quad x \in \Omega \\
z(t, x)=0, \quad t \geq 0, \quad x \in \partial \Omega
\end{array}\right.
$$

is approximately controllable.

After that, we write the system(1.1) as follows

$$
\left\{\begin{array}{l}
z_{t}-a \Delta z_{t}-b \Delta z=1_{\omega} u(t, x)+e z+c u(t, x)+g(t, z, u(t, x)), t \in(0, \tau], x \in \Omega \\
z(t, x)=0, \quad t \geq 0, \quad x \in \partial \Omega
\end{array}\right.
$$

where $g(t, z, u)=f(t, z, u)-e z-c u$ is an smooth and bounded function.

The technique we use here to prove the controllability of the linear equation (1.7) is based in the following results:

Theorem 1.1. (see Theorem 1.23 from [2], p. 20) Suppose $\Omega \subset \mathbb{R}^{n}$ is open, nonempty and connected set, and $f$ is real analytic function in $\Omega$ with $f=0$ on a non-empty open subset $\omega$ of $\Omega$. Then, $f=0$ in $\Omega$.

Lemma 1.1. (see Lemma 3.14 from [4], p. 62)Let $\left\{\alpha_{j}\right\}_{j \geq 1}$ and $\left\{\beta_{i, j}: i=1,2, \ldots, m\right\}_{j \geq 1}$ be sequences of real numbers such that: $\alpha_{1}>\alpha_{2}>\alpha_{3} \cdots$. Then

$$
\sum_{j=1}^{\infty} e^{\alpha_{j} t} \beta_{i, j}=0, \quad \forall t \in\left[0, t_{1}\right], \quad i=1,2, \cdots, m
$$

if and only if

$$
\beta_{i, j}=0, \quad i=1,2, \cdots, m ; j=1,2, \cdots, \infty \text {. }
$$

Finally, the approximate controllability of the system (1.8) follows from the controllability of (1.7) and Schauder fixed point Theorem.

\section{Abstract Formulation of the Problem.}

In this section we describe the space in which this problem will be situated as an abstract ordinary differential equation. 
Let $Z=L^{2}(\Omega)=L^{2}(\Omega, \mathbb{R})$ and consider the linear unbounded operator $A$ : $D(A) \subset Z \rightarrow Z$ defined by $A \phi=-\Delta \phi$, where

$$
D(A)=H^{2}(\Omega, \mathbb{R}) \cap H_{0}^{1}(\Omega, \mathbb{R}) .
$$

The operator $A$ has the following very well known properties: the spectrum of $A$ consists of eigenvalues

$$
0<\lambda_{1}<\lambda_{2}<\cdots<\lambda_{j}<\cdots \quad \text { with } \quad \lambda_{j} \rightarrow \infty,
$$

each one with finite multiplicity $\gamma_{j}$ equal to the dimension of the corresponding eigenspace. Therefore:

a) There exists a complete orthonormal set $\left\{\phi_{j, k}\right\}$ of eigenvectors of $\mathrm{A}$.

b) For all $z \in D(A)$ we have

$$
A z=\sum_{j=1}^{\infty} \lambda_{j} \sum_{k=1}^{\gamma_{j}}<z, \phi_{j, k}>\phi_{j, k}=\sum_{j=1}^{\infty} \lambda_{j} E_{j} z,
$$

where $\langle\cdot, \cdot\rangle$ is the inner product in $Z$ and

$$
E_{j} z=\sum_{k=1}^{\gamma_{j}}<z, \phi_{j, k}>\phi_{j, k}
$$

So, $\left\{E_{j}\right\}$ is a family of complete orthogonal projections in $Z$ and

$$
z=\sum_{j=1}^{\infty} E_{j} z, \quad z \in Z
$$

c) $-A$ generates the analytic semigroup $\left\{e^{-A t}\right\}$ given by

$$
e^{-A t} z=\sum_{j=1}^{\infty} e^{-\lambda_{j} t} E_{j} z
$$

Consequently, systems (1.1), (1.7) and (1.8) can be written respectively as abstract differential equations in $Z$ :

$$
\begin{aligned}
& z^{\prime}+a A z^{\prime}+b A z=1_{\omega} u(t)+f^{e}(t, z, u), \quad z \in Z \quad t \in(0, \tau], \\
& z^{\prime}+a A z^{\prime}+b A z=1_{\omega} u(t)+e z+c u, \quad z \in Z \quad t \in(0, \tau], \\
& z^{\prime}+a A z^{\prime}+b A z=1_{\omega} u(t)+e z+c u+g^{e}(t, z, u), \quad z \in Z \quad t \in(0, \tau],
\end{aligned}
$$

where $u \in L^{2}([0, \tau] ; U), U=Z, B_{\omega}: U \longrightarrow Z, B_{\omega} u=1_{\omega} u$ is a bounded linear operator, $f^{e}:[0, \tau] \times Z \times U \rightarrow Z$ is defined by $f^{e}(t, z, u)(x)=f(t, z(x), u(x)), \quad \forall x \in \Omega$ and $g^{e}(t, z, u)=f^{e}(t, z, u)-e z-c u$. On the other hand, the hypothesis (1.2) implies that

$$
\sup _{(t, z, u) \in Z_{\tau}}\left\|f^{e}(t, z, u)-e z-c u\right\|_{Z}<\infty
$$


where $Z_{\tau}=[0, \tau] \times Z \times U$. Therefore, $g^{e}:[0, \tau] \times Z \times U \rightarrow Z$ is bounded and smooth enough.

Since $(I+a A)=a\left(A-\left(-\frac{1}{a}\right) I\right)$ and $-\frac{1}{a} \in \rho(A)(\rho(A)$ is the resolvent set of $A)$, then the operator:

$$
I+a A: D(A) \rightarrow Z
$$

is invertible with bounded inverse

$$
(I+a A)^{-1}: Z \rightarrow D(A)
$$

Therefore, equations $(2.6),(2.7)$ and (2.8) also can be written as follows

$$
\begin{aligned}
z^{\prime}+b(I+a A)^{-1} A z= & (I+a A)^{-1} 1_{\omega} u(t) \\
& +(I+a A)^{-1} f^{e}(t, z, u), \quad z \in Z, \quad t \in(0, \tau] . \\
z^{\prime}+b(I+a A)^{-1} A z= & (I+a A)^{-1} 1_{\omega} u(t)+e(I+a A)^{-1} z \\
& +c(I+a A)^{-1} u, \quad z \in Z, \quad t \in(0, \tau] . \\
z^{\prime}+b(I+a A)^{-1} A z= & (I+a A)^{-1} 1_{\omega} u(t)+e(I+a A)^{-1} z \\
& +c(I+a A)^{-1} u+(I+a A)^{-1} g^{e}(t, z, u), \quad z \in Z, \quad t \in(0, \tau] .
\end{aligned}
$$

Moreover, $(I+a A)$ and $(I+a A)^{-1}$ can be written in terms of the eigenvalues of A:

$$
\begin{gathered}
(I+a A) z=\sum_{j=1}^{\infty}\left(1+a \lambda_{j}\right) E_{j} z \\
(I+a A)^{-1} z=\sum_{j=1}^{\infty} \frac{1}{1+a \lambda_{j}} E_{j} z .
\end{gathered}
$$

Therefore, if we put $B=(I+a A)^{-1}$ and $F(t, z, u)=(I+a A)^{-1} f^{e}(t, z, u)$, equations $(2.10),(2.11)$ and $(2.12)$ can be written in the form:

$$
\begin{gathered}
z^{\prime}+b B A z=B B_{\omega} u(t)+F(t, z, u), \quad t \in(0, \tau], \\
z^{\prime}+b B A z=B B_{\omega} u(t)+e B z+c B u, \quad t \in(0, \tau], \\
z^{\prime}+b B A z=B B_{\omega} u(t)+e B z+c B u+G(t, z, u), \quad t \in(0, \tau],
\end{gathered}
$$

where $B_{\omega} f=1_{\omega} f$ is a linear a bounded operator from $Z$ to $Z$ and $u \in L^{2}\left(0, \tau ; L^{2}(\Omega)\right)$ $=L^{2}(0, \tau ; Z)$ and $G(t, z, u)=F(t, z, u)-e B z-c B u$ is smooth enough and bounded. Now, we formulate two simple propositions.

Proposition 2.1. ([11]) The operators $b B A$ and $T(t)=e^{-b B A t}$ are given by the following expressions

$$
b B A z=\sum_{j=1}^{\infty} \frac{b \lambda_{j}}{1+a \lambda_{j}} E_{j} z
$$




$$
T_{b}(t) z=e^{-b B A t} z=\sum_{j=1}^{\infty} e^{\frac{-b \lambda_{j}}{1+a \lambda_{j}} t} E_{j} z .
$$

Moreover, the following estimate holds

$$
\|T(t)\| \leq e^{-\beta t}, \quad t \geq 0
$$

where

$$
\beta=\inf _{j \geq 1}\left\{\frac{b \lambda_{j}}{1+a \lambda_{j}}\right\}=\frac{b \lambda_{1}}{1+a \lambda_{1}} .
$$

Observe that, due to the above notation, the system (2.14) can be written as follows

$$
z^{\prime}=-\mathcal{A} z+B B_{\omega} u(t)+F(t, z, u), \quad t \in(0, \tau]
$$

where $\mathcal{A}=b B A$.

Proposition 2.2. The operators $e B-\mathcal{A}$ and $T_{e}(t)=e^{(e B-\mathcal{A}) t}$ are given by the following expressions

$$
\begin{gathered}
(e B-\mathcal{A}) z=\sum_{j=1}^{\infty} \frac{e-b \lambda_{j}}{1+a \lambda_{j}} E_{j} z \\
T_{e}(t) z=e^{(e B-\mathcal{A}) t} z=\sum_{j=1}^{\infty} e^{\frac{e-b \lambda_{j}}{1+a \lambda_{j}} t} E_{j} z,
\end{gathered}
$$

and

$$
\left\|T_{e}(t)\right\| \leq e^{\rho t}, \quad t \geq 0
$$

where

$$
\rho=\frac{e-b \lambda_{1}}{1+a \lambda_{1}}
$$

provided that $b+e a>0$.

Notice that systems (2.15) and (2.16) can be written in the form:

$$
\begin{gathered}
z^{\prime}=(e B-\mathcal{A}) z+B B_{\omega} u(t)+c B u, \quad t \in(0, \tau] \\
z^{\prime}=(e B-\mathcal{A}) z+B B_{\omega} u(t)+c B u+G(t, z, u), \quad t \in(0, \tau] .
\end{gathered}
$$

\section{Controllability of the Auxiliary Linear Equation (1.7)}

In this section we prove the interior controllability of the linear system (2.26). But, at the beginning we give the definition of approximate controllability for this system. To this end, notice that for an arbitrary $z_{0} \in Z$ and $u \in L^{2}(0, \tau ; U)$ the initial value problem

$$
\left\{\begin{array}{l}
z^{\prime}=(e B-\mathcal{A}) z+B B_{\omega} u(t)+c B u, \quad t \in(0, \tau] \\
z(0)=z_{0}
\end{array}\right.
$$


where the control function $u$ belong to $L^{2}(0, \tau ; U)$, admits only one mild solution given by

$$
z(t)=T_{e}(t) z_{0}+\int_{0}^{t} T_{e}(t-s)\left(B B_{\omega}+c B I\right) u(s) d s, \quad t \in[0, \tau] .
$$

Definition 3.1. (Approximate Controllability) The system (2.26) is said to be approximately controllable on $[0, \tau]$ if for every $z_{0}, z_{1} \in Z, \varepsilon>0$ there exists $u \in$ $L^{2}(0, \tau ; U)$ such that the solution $z(t)$ of (3.2) corresponding to $u$ verifies:

$$
\left\|z(\tau)-z_{1}\right\|<\varepsilon .
$$

Definition 3.2. For the system (2.26) we define the following concept: The controllability map (for $\tau>0) G_{\mathbf{e}}: L^{2}(0, \tau ; U) \longrightarrow Z$ is given by

$$
G_{\mathbf{e}} u=\int_{0}^{\tau} T_{e}(s)\left(B B_{\omega}+c B I\right) u(s) d s,
$$

whose adjoint operator $G_{\mathbf{e}}^{*}: Z \longrightarrow L^{2}(0, \tau ; Z)$ is given by

$$
\left(G_{\mathbf{e}}^{*} z\right)(s)=\left(B_{\omega}^{*}+c I\right) B^{*} T_{e}^{*}(s) z, \quad \forall s \in[0, \tau], \quad \forall z \in Z .
$$

The following lemma holds in general for a linear bounded operator $G: W \rightarrow Z$ between Hilbert spaces $W$ and $Z$.

Lemma 3.1. (see [4], [5], [1] and [14]) The equation (2.26) is approximately controllable on $[0, \tau]$ if and only if one of the following statements holds:

a) $\overline{\operatorname{Rang}\left(G_{\mathbf{e}}\right)}=Z$.

b) $\operatorname{Ker}\left(G_{\mathbf{e}}^{*}\right)=\{0\}$.

c) $\left\langle G_{\mathbf{e}} G_{\mathbf{e}}^{*} z, z\right\rangle>0, z \neq 0$ in $Z$.

d) $\lim _{\alpha \rightarrow 0^{+}} \alpha\left(\alpha I+G_{\mathbf{e}} G_{\mathbf{e}}^{*}\right)^{-1} z=0$.

e) $\sup _{\alpha>0}\left\|\alpha\left(\alpha I+G_{\mathbf{e}} G_{\mathbf{e}}^{*}\right)^{-1}\right\| \leq 1$.

f) $\left(B_{\omega}^{*}+e I\right) B^{*} T_{e}^{*}(t) z=0, \quad \forall t \in[0, \tau], \quad \Rightarrow z=0$.

g) For all $z \in Z$ we have $G_{e} u_{\alpha}=z-\alpha\left(\alpha I+G_{\mathbf{e}} G_{\mathbf{e}}^{*}\right)^{-1} z$, where

$$
u_{\alpha}=G_{\mathbf{e}}^{*}\left(\alpha I+G_{\mathbf{e}} G_{\mathbf{e}}^{*}\right)^{-1} z, \quad \alpha \in(0,1] .
$$

So, $\lim _{\alpha \rightarrow 0} G_{\mathbf{e}} u_{\alpha}=z$ and the error $E_{\alpha} z$ of this approximation is given by

$$
E_{\alpha} z=\alpha\left(\alpha I+G_{\mathbf{e}} G_{\mathbf{e}}^{*}\right)^{-1} z, \quad \alpha \in(0,1] .
$$


Remark 3.1. The Lemma 3.1 implies that the family of linear operators $\Gamma_{\alpha}: Z \rightarrow$ $L^{2}(0, \tau ; U)$, defined for $0<\alpha \leq 1$ by

$$
\Gamma_{\alpha} z=\left(B_{\omega}^{*}+e I\right) B^{*} T_{e}^{*}(\cdot)\left(\alpha I+G_{\mathbf{e}} G_{\mathbf{e}}^{*}\right)^{-1} z=G_{\mathbf{e}}^{*}\left(\alpha I+G_{\mathbf{a}} G_{\mathbf{e}}^{*}\right)^{-1} z,
$$

is an approximate inverse for the right of the operator $G_{\mathbf{a}}$ in the sense that

$$
\lim _{\alpha \rightarrow 0} G_{\mathbf{e}} \Gamma_{\alpha}=I
$$

Theorem 3.1. The system (2.7) is approximately controllable on $[0, \tau]$. Moreover, a sequence of controls steering the system (2.7) from initial state $z_{0}$ to an $\epsilon$ neighborhood of the final state $z_{1}$ at time $\tau>0$ is given by the formula

$$
u_{\alpha}(t)=\left(B_{\omega}^{*}+e I\right) B^{*} T_{e}^{*}(t)\left(\alpha I+G_{\mathbf{e}} G_{\mathbf{e}}^{*}\right)^{-1}\left(z_{1}-T_{e}(\tau) z_{0}\right),
$$

and the error of this approximation $E_{\alpha}$ is given by the expresion

$$
E_{\alpha}=\alpha\left(\alpha I+G_{\mathbf{e}} G_{\mathbf{e}}^{*}\right)^{-1}\left(z_{1}-T(\tau) z_{0}\right) .
$$

Proof . It is enough to show that the restriction $G_{\mathbf{e}, \omega}=\left.G_{\mathbf{e}}\right|_{L^{2}\left(0, \tau ; L^{2}(\omega)\right)}$ of $G_{\mathbf{e}}$ to the space $L^{2}\left(0, \tau ; L^{2}(\omega)\right)$ has range dense, i.e., $\overline{\operatorname{Rang}\left(G_{\mathbf{e}, \omega}\right)}=Z$ or $\operatorname{Ker}\left(G_{\mathbf{e}, \omega}^{*}\right)=\{0\}$. Consequently, $G_{\mathbf{a}, \omega}: L^{2}\left(0, \tau ; L^{2}(\omega)\right) \rightarrow Z$ takes the following form

$$
G_{\mathbf{e}, \omega} u=\int_{0}^{\tau} T_{e}(s) B(1+c) u(s) d s .
$$

whose adjoint operator $G_{\mathbf{e}, \omega}^{*}: Z \longrightarrow L^{2}\left(0, \tau ; L^{2}(\omega)\right)$ is given by

$$
\left(G_{\mathbf{e}, \omega} z\right)(s)=(1+c) B^{*} T_{e}^{*}(s) z, \quad \forall s \in[0, \tau], \quad \forall z \in Z .
$$

Since $B$ is given by the formula

$$
B z=\sum_{j=1}^{\infty} \frac{1}{1+a \lambda_{j}} E_{j} z,
$$

and $T_{e}$ by $(2.23)$, we get that $B=B^{*}$ and $T_{e}^{*}(t)=T_{e}$.

Suppose that

$$
(1+c) B^{*} T_{e}^{*}(t) z=0, \quad \forall t \in[0, \tau] .
$$

Since $1+c \neq 0$, this is equivalents to the equality

$$
B^{*} T_{e}^{*}(t) z=0, \quad \forall t \in[0, \tau] .
$$

On the other hand, we have

$$
B^{*} T_{e}^{*}(t) z=\sum_{j=1}^{\infty} \frac{e^{-\gamma_{j} t}}{1+a \lambda_{j}} E_{j} z=0
$$


where $\gamma_{j}=\frac{b \lambda_{j}-e}{1+a \lambda_{j}}$, which satisfies the conditions:

$$
0<\gamma_{1}<\gamma_{2}<\cdots<\gamma_{j}<\cdots
$$

Hence, following the proof of Lemma 1.1, we obtain that

$$
E_{j} z(x)=\sum_{k=1}^{\gamma_{j}}<z, \phi_{j, k}>\phi_{j, k}(x)=0, \quad \forall x \in \omega, \quad j=1,2,3, \ldots
$$

Since $\phi_{j, k}$ are analytic functions on $\Omega$, from Theorem 1.1 we obtain that

$$
E_{j} z(x)=\sum_{k=1}^{\gamma_{j}}<z, \phi_{j, k}>\phi_{j, k}(x)=0, \quad \forall x \in \Omega, \quad j=1,2,3, \ldots
$$

Therefore, $E_{j} z=0, \quad j=1,2,3, \ldots$, which implies that $z=0$. So, $\overline{\operatorname{Rang}\left(G_{\mathbf{e}, \omega}\right)}=$ $Z$, and consequently $\overline{\operatorname{Rang}\left(G_{\mathbf{e}}\right)}=Z$. Hence, the system (2.26) is approximately controllable on $[0, \tau]$, and the remainder of the proof follows from Lemma 3.1.

\section{Controllability of the Semilinear BBM Equation}

In this section we prove the main result of this paper, the interior controllability of the semilinear BBM Equation given by (1.1), which is equivalent to prove the approximate controllability of the system (2.27). To this end, observe that for all $z_{0} \in Z$ and $u \in L^{2}(0, \tau ; U)$ the initial value problem

$$
\left\{\begin{array}{l}
z^{\prime}=(e B-\mathcal{A}) z+B B_{\omega} u(t)+c B u+G(t, z, u), \quad t \in(0, \tau], \\
z(0)=z_{0}
\end{array}\right.
$$

where the control function $u$ belongs to $L^{2}(0, \tau ; U)$, admits only one mild solution given by the formula

$$
\begin{aligned}
z_{u}(t) & =T_{e}(t) z_{0}+\int_{0}^{t} T_{e}(t-s)\left(B B_{\omega}+c B I\right) u(s) d s \\
& +\int_{0}^{t} T_{e}(t-s) G\left(s, z_{u}(s),(s)\right) d s, \quad t \in[0, \tau]
\end{aligned}
$$

Definition 4.1. (Approximate Controllability) The system (2.27) is said to be approximately controllable on $[0, \tau]$ if for every $z_{0}, z_{1} \in Z, \varepsilon>0$ there exists $u \in$ $L^{2}(0, \tau ; U)$ such that the solution $z(t)$ of (4.2) corresponding to $u$ verifies

$$
\left\|z(\tau)-z_{1}\right\|<\varepsilon
$$


Definition 4.2. For the system (2.27) we define the following concept: The nonlinear controllability map (for $\tau>0) G_{g}: L^{2}(0, \tau ; U) \longrightarrow Z$ is given by the formula

$G_{g} u=\int_{0}^{\tau} T_{e}(s)\left(B B_{\omega}+c B I\right) u(s) d s+\int_{0}^{\tau} T_{e}(s) G\left(s, z_{u}(s), u(s)\right) d s=G_{e}(u)+H(u)$,

where $H: L^{2}(0, \tau ; U) \longrightarrow Z$ is the nonlinear operator given by

$$
H(u)=\int_{0}^{\tau} T_{e}(s) G\left(s, z_{u}(s), u(s)\right) d s, \quad u \in L^{2}(0, \tau ; U)
$$

The following lemma is trivial.

Lemma 4.1. The equation (2.27) is approximately controllable on $[0, \tau]$ if and only if $\overline{\operatorname{Rang}\left(G_{g}\right)}=Z$.

Definition 4.3. The following equation

$$
u_{\alpha}=\Gamma_{\alpha}\left(z-H\left(u_{\alpha}\right)\right)=G_{e}^{*}\left(\alpha I+G_{e} G_{e}^{*}\right)^{-1}\left(z-H\left(u_{\alpha}\right)\right), \quad(0<\alpha \leq 1),
$$

will be called the controllability equations associated to the non linear equation (2.27).

Now, we are ready to present and prove the main result of this paper, which is the interior approximate controllability of the semilinear BBM equation (1.1), and for the proof we will use some ideas from Propositions 4.2 from [1].

Theorem 4.1. If the operator $H$ define by (4.4) is compact and $\overline{\operatorname{Rang}(H)}$ is compact set, then the system (2.27) is approximately controllable on $[0, \tau]$. Moreover, a sequence of controls steering the system (2.27) from initial state $z_{0}$ to an $\epsilon$ neighborhood of the final state $z_{1}$ at time $\tau>0$ is given by the formula

$$
u_{\alpha}(t)=\left(B_{\omega}^{*}+e I\right) B^{*} T_{e}^{*}(t)\left(\alpha I+G_{e} G_{e}^{*}\right)^{-1}\left(z_{1}-T(\tau) z_{0}-H\left(u_{\alpha}\right)\right),
$$

and the error of this approximation $E_{\alpha}$ is given by the

$$
E_{\alpha}=\alpha\left(\alpha I+G_{e} G_{e}^{*}\right)^{-1}\left(z_{1}-T(\tau) z_{0}-H\left(u_{\alpha}\right)\right) .
$$

Proof For each fixed $z \in Z$ we consider the following family of nonlinear operators $K_{\alpha}: L^{2}(0, \tau ; U) \rightarrow L^{2}(0, \tau ; U)$, given by the formula

$$
K_{\alpha}(u)=\Gamma_{\alpha}(z-H(u))=G_{e}^{*}\left(\alpha I+G_{e} G_{e}^{*}\right)^{-1}(z-H(u)), \quad(0<\alpha \leq 1) .
$$

First, we prove that, for all $\alpha \in(0,1]$ the operator $K_{\alpha}$ has a fixed point $u_{\alpha}$. In fact, since the operator $H$ is a compact operator, then the operator $K_{\alpha}$ is compact. On the other hand, since $G(t, z, u)$ is bounded and $\left\|T_{e}(t)\right\| \leq R e^{W t}, \quad t \geq 0$, there exists a constant $M>0$ such that

$$
\|H(u)\| \leq M, \quad \forall u \in L^{2}(0, \tau ; U) .
$$


Then,

$$
\left\|K_{\alpha}(u)\right\| \leq\left\|\Gamma_{\alpha}\right\|(\|z\|+M), \quad \forall u \in L^{2}(0, \tau ; U) .
$$

Therefore, the operator $K_{\alpha}$ maps the ball $B_{r}(0) \subset L^{2}(0, \tau ; U)$ of center zero and radio $r \geq\left\|\Gamma_{\alpha}\right\|(\|z\|+M)$ into itself. Hence, applying the Schauder fixed point Theorem we get that the operator $K_{\alpha}$ has a fixed point $u_{\alpha} \in B_{r}(0) \subset L^{2}(0, \tau ; U)$.

Since $\overline{\operatorname{Rang}(H)}$ is compact, without loss of generality, we can assume that the sequence $H\left(u_{\alpha}\right)$ converges to $y \in Z$. So,

$$
u_{\alpha}=\Gamma_{\alpha}\left(z-H\left(u_{\alpha}\right)\right)=G_{e}^{*}\left(\alpha I+G_{e} G_{e}^{*}\right)^{-1}\left(z-H\left(u_{\alpha}\right)\right) .
$$

Then, we get

$$
\begin{aligned}
G_{e} u_{\alpha} & =G_{e} \Gamma_{\alpha}\left(z-H\left(u_{\alpha}\right)\right)=G_{e} G_{e}^{*}\left(\alpha I+G_{e} G_{e}^{*}\right)^{-1}\left(z-H\left(u_{\alpha}\right)\right) \\
& =\left(\alpha I+G_{e} G_{e}^{*}-\alpha I\right)\left(\alpha I+G_{e} G_{e}^{*}\right)^{-1}\left(z-H\left(u_{\alpha}\right)\right) \\
& =z-H\left(u_{\alpha}\right)-\alpha\left(\alpha I+G_{e} G_{e}^{*}\right)^{-1}\left(z-H\left(u_{\alpha}\right)\right)
\end{aligned}
$$

Hence, we deduce the following equality

$$
G_{e} u_{\alpha}+H\left(u_{\alpha}\right)=z-\alpha\left(\alpha I+G_{e} G_{e}^{*}\right)^{-1}\left(z-H\left(u_{\alpha}\right)\right) .
$$

To conclude the proof, it enough to prove that

$$
\lim _{\alpha \rightarrow 0}\left\{-\alpha\left(\alpha I+G_{e} G_{e}^{*}\right)^{-1}\left(z-H\left(u_{\alpha}\right)\right)\right\}=0
$$

From Lemma $3.1 \mathrm{~d}$ ) we get that

$$
\begin{aligned}
\lim _{\alpha \rightarrow 0}\left\{-\alpha\left(\alpha I+G_{e} G_{e}^{*}\right)^{-1}\left(z-H\left(u_{\alpha}\right)\right)\right\} & =-\lim _{\alpha \rightarrow 0}\left\{-\alpha\left(\alpha I+G_{e} G_{e}^{*}\right)^{-1} H\left(u_{\alpha}\right)\right\} \\
=-\lim _{\alpha \rightarrow 0}-\alpha\left(\alpha I+G_{e} G_{e}^{*}\right)^{-1} y & -\lim _{\alpha \rightarrow 0}-\alpha\left(\alpha I+G_{e} G_{e}^{*}\right)^{-1}\left(H\left(u_{\alpha}\right)-y\right) \\
& =\lim _{\alpha \rightarrow 0}-\alpha\left(\alpha I+G_{e} G_{e}^{*}\right)^{-1}\left(H\left(u_{\alpha}\right)-y\right) .
\end{aligned}
$$

On the other hand, from Lemma $3.1 \mathrm{e}$, we obtain that

$$
\left\|\alpha\left(\alpha I+G G^{*}\right)^{-1}\left(H\left(u_{\alpha}\right)-y\right)\right\| \leq\left\|\left(H\left(u_{\alpha}\right)-y\right)\right\| .
$$

Therefore, keeping in mind that $H\left(u_{\alpha}\right)$ converges to $y$, we conclude that

$$
\lim _{\alpha \rightarrow 0}\left\{-\alpha\left(\alpha I+G G^{*}\right)^{-1}\left(z-H\left(u_{\alpha}\right)\right)\right\}=0
$$

So, putting $z=z_{1}-T_{e}(\tau) z_{0}$ and using (4.2), we obtain the desired result

$$
\begin{aligned}
z_{1}= & \lim _{\alpha \rightarrow 0^{+}}\left\{T_{e}(\tau) z_{0}+\int_{0}^{\tau} T_{e}(\tau-s)\left(B B_{\omega}+c B I\right) u_{\alpha}(s) d s\right. \\
& \left.+\int_{0}^{\tau} T_{e}(\tau-s) G\left(s, z_{u_{\alpha}}(s), u_{\alpha}(s)\right) d s\right\}
\end{aligned}
$$

Remark 4.1. In the particular case that $a=0$ and $b=1$ the operator $H$ define by (4.4) is compact and $\overline{\operatorname{Rang}(H)}$ is compact set (see [3]), and as a consequence we obtain the interior approximate controllability of the semilinear heat equation (see [12]). 


\section{Final Remark}

Our technique is simple and can be applied to those system involving diffusion process like some control system governed by heat equations. For example, the strongly damped wave equations, beam equations and so on.

Let us provide these two examples where this technique may be used.

Example 5.1. Notice that this technique can be applied to prove the interior controllability of the strongly damped wave equation with Dirichlet boundary conditions

$$
\begin{cases}w_{t t}+\eta(-\Delta)^{1 / 2} w_{t}+\gamma(-\Delta) w=1_{\omega} u(t, x)+f(t, z, u(t)), & \text { in }(0, \tau] \times \Omega \\ w=0, & \text { in }(0, \tau] \times \partial \Omega \\ w(0, x)=w_{0}(x), \quad w_{t}(0, x)=w_{1}(x), & \text { in } \Omega\end{cases}
$$

in the space $Z_{1 / 2}=D\left((-\Delta)^{1 / 2}\right) \times L^{2}(\Omega)$, where $\Omega$ is a bounded domain in $\mathbb{R}^{n}$, $\omega$ is an open nonempty subset of $\Omega, 1_{\omega}$ denotes the characteristic function of the set $\omega$, the distributed control $u \in L^{2}\left(0, \tau ; L^{2}(\Omega)\right)$ and $\eta, \gamma$ are positive numbers.

Example 5.2. Another example, where this technique may be applied, is the partial differential equations modeling the structural damped vibrations of a string or a beam having the form

$$
\begin{cases}y_{t t}-2 \beta \Delta y_{t}+\Delta^{2} y=1_{\omega} u(t, x)+f(t, z, u(t)), & \text { on } \quad(0, \tau] \times \Omega \\ y=\Delta y=0, & \text { on } \quad(0, \tau] \times \partial \Omega \\ y(0, x)=y_{0}(x), \quad y_{t}(0, x)=y_{1}(x), & \text { in } \quad \Omega\end{cases}
$$

where $\Omega$ is a bounded domain in $\mathbb{R}^{n}, \omega$ is an open nonempty subset of $\Omega, 1_{\omega}$ denotes the characteristic function of the set $\omega$, the distributed control $u \in L^{2}\left(0, \tau ; L^{2}(\Omega)\right)$ and $y_{0}, y_{1} \in L^{2}(\Omega)$.

\section{References}

[1] J. Appel, H.Leiva, N. Merentes, A. Vignoli, Un espectro de compresión no lineal con aplicaciones a la controlabilidad aproximada de sistemas semilineales, preprint

[2] S. Axler, P. Bourdon and W. Ramey, Harmonic Fucntion Theory. Graduate Texts in Math., 137. Springer Verlag, New York (1992).

[3] D. Barcenas, H. Leiva AND Z. Sivoli, A Broad Class of Evolution Equations are Approximately Controllable, but Never Exactly Controllable. IMA J. Math. Control Inform. 22, no. 3 (2005), 310-320.

[4] R.F. Curtain, A.J. Pritchard, Infinite Dimensional Linear Systems. Lecture Notes in Control and Information Sciences, 8. Springer Verlag, Berlin (1978).

[5] R.F. Curtain, H.J. Zwart, An Introduction to Infinite Dimensional Linear Systems Theory. Text in Applied Mathematics, 21. Springer Verlag, New York (1995).

[6] J.I. DIAZ, J.Henry and A.M. Ramos, On the Approximate Controllability of Some Semilinear Parabolic Boundary-Value Problemas, Appl. Math. Optim 37-71 (1998). 
[7] E. Fernandez-Cara, Remark on Approximate and Null Controllability of Semilinear Parabolic Equations ESAIM:Proceeding OF CONTROLE ET EQUATIONS AUX DERIVEES PARTIELLES, Vol.4, 1998, 73-81.

[8] E. Fernandez-Cara and E. Zuazua, Controllability for Blowing up Semilinear Parabolic Equations, C.R. Acad. Sci. Paris, t. 330, serie I, p. 199-204, 2000.

[9] Luiz A. F. de Oliveira, On Reaction-Diffusion Systems E. Journal of Differential Equations, Vol. 1998(1998), N0. 24, pp. 1-10.

[10] H. Leiva, A Lemma on $C_{0}$-Semigroups and Applications PDEs Systems, Quaestions Mathematicae, Vol. 26, pp. 247-265 (2003).

[11] H. Leiva, N. Merentes and J.L. Sanchez, Interior Controllability of the Benjamin-BonaMahony Equation, Journal of Mathematis and Applications, No 33,pp. 51-59 (2010).

[12] H. H. Leiva, N. Merentes and J.L. Sanchez, Interior Controllability of the nD Semilinear Heat Equation, African Diaspora of Mathematics. Spetial Volume in Honor to Profs. C. Carduneanu, A. Fink, and S. Zaideman. Vol. 12, Number 2, pp. 1-12(2011).

[13] H. Leiva, Controllability of a System of Parabolic equation with non-diagonal diffusion matrix. IMA Journal of Mathematical Control and Information; Vol. 32, 2005, pp. 187199.

[14] H. Leiva and Y. Quintana, Interior Controllability of a Broad Class of Reaction Diffusion Equations, Mathematical Problems in Engineering, Vol. 2009, Article ID 708516, 8 pages, doi:10.1155/2009/708516.

[15] Xu Zhang, A Remark on Null Exact Controllability of the Heat Equation. IAM J. CONTROL OPTIM. Vol. 40, No. 1(2001), pp. 39-53.

[16] E. Zuazua, Controllability of a System of Linear Thermoelasticity, J. Math. Pures Appl., 74, (1995), 291-315.

[17] E. Zuazua, Control of Partial Differential Equations and its Semi-Discrete Approximation. Discrete and Continuous Dynamical Systems, vol. 8, No. 2. April (2002), 469-513.

DOI: $10.7862 / \mathrm{rf} .2012 .4$

\section{Hugo Leiva}

email: hleiva@ula.ve

Departamento de Matemáticas

Universidad de Los Andes

Mérida 5101- VENEZUELA

Nelson Merentes

email: nmerucv@gmail.com

José L. Sánchez

Departamento de Matemáticas

Facultad de Ciencias

Universidad Central de Venezuela

Caracas 1051 VENEZUELA

Received 10.10.2011 